\title{
MR Imaging of the Facial Nerve through the Temporal Bone at 3T with a Noncontrast Ultrashort Echo Time Sequence
}

\author{
(D).P. Guenette, (DR.T. Seethamraju, (D). Jayender, (D)C.E. Corrales, and (D)T.C. Lee
}

\begin{abstract}
SUMMARY: The pointwise encoding time reduction with radial acquisition (PETRA) ultrashort echo time MR imaging sequence at 3T enables visualization of the facial nerve from the brain stem, through the temporal bone, to the stylomastoid foramen without intravenous contrast. Use of the PETRA sequence, or other ultrashort echo time sequences, should be considered in the MR imaging evaluation of certain skull base tumors and perhaps other facial nerve and temporal bone pathologies.
\end{abstract}

ABBREVIATIONS: CNR = contrast-to-noise ratio; IR-FSPGR = inversion recovery-prepared fast spoiled gradient recalled-echo; PETRA = pointwise encoding time reduction with radial acquisition; UTE $=$ ultrashort echo time

T he cisternal, canalicular, labyrinthine, geniculate, tympanic, and mastoid segments of the facial nerve are either not detectable or only faintly visible on noncontrast T1-weighted MR images. ${ }^{1}$ Moreover, although contrast enhancement of the surrounding venous plexus can aid in facial nerve visualization, the extent of enhancement is variable and unreliable. ${ }^{2}$ We hypothesized that visibility of the facial nerve could be improved with ultrashort echo time (UTE) imaging. UTE sequences capture signal from rapidly decaying short-T2 tissue, such as cortical bone ${ }^{3}$ and middle ear ossicles ${ }^{4}$ and, therefore, should capture signal from the petrous and mastoid portions of the temporal bone. Moreover, peripheral nerves have detectable signal in the ultrashort T2 spectrum, ${ }^{5}$ so UTE imaging should provide visualization of the facial nerve. Finally, UTE imaging minimizes air-related susceptibility artifacts ${ }^{6}$ and would therefore minimize artifacts from the middle ear cavity and mastoid air cells that may contribute to nonvisualization of the facial nerve on spin-echo and gradient-echo sequences. The pointwise encoding time reduction with radial acquisition (PETRA) UTE sequence provides more con-

Received April 25, 2018; accepted after revision June 3.

From the Division of Neuroradiology (J.P.G., J.J., T.C.L.) and Division of Otolaryngology-Head and Neck Surgery (C.E.C.), Brigham and Women's Hospital, Harvard Medical School, Boston, Massachusetts; and Siemens Medical Solutions USA, Inc. (R.T.S.), Boston, Massachusetts.

This study was supported by the American Society of Head and Neck Radiology through the 2017 William N. Hanafee Research Grant. Jagadeesan Jayender was supported by the National Institute of Biomedical Imaging and Bioengineering, National Institutes of Health, through grant number P41EB015898.

Please address correspondence to Jeffrey P. Guenette, MD, Division of Neuroradiology, Brigham and Women's Hospital, 75 Francis St, Boston, MA 02135; e-mail: jpguenette@bwh.harvard.edu; @gunetty

- Indicates open access to non-subscribers at www.ajnr.org

http://dx.doi.org/10.3174/ajnr.A5754 sistent image quality over a wider range of conditions than similar sequences. ${ }^{7}$ This report describes the evaluation of the facial nerve segments through the temporal bone using both the default PETRA protocol and a shortened 4-minute PETRA protocol.

\section{MATERIALS AND METHODS}

As part of a prospective study designed to evaluate the extracranial facial nerve, an MR imaging examination of the brain and face that included a PETRA sequence was performed in 8 healthy subjects ( 6 men, 2 women; $31 \pm 8$ years of age) between January and March 2018. All subjects provided informed consent. The study was approved by our institutional review board and performed in compliance with the Health Insurance Portability and Accountability Act.

\section{Imaging Protocols}

All imaging was performed on a Magnetom Prisma 3T MR imaging system with a 64-channel head/neck coil (Siemens, Erlangen, Germany). The default PETRA protocol as installed by Siemens (Table 1) was performed on 7 subjects and 14 facial nerves. Due to the 6-minute duration of the protocol, parameter modifications were tested to reduce imaging time while retaining the signal-to-noise ratio and contrast-to-noise ratio (CNR) of the facial nerve and surrounding structures. A modified 4-minute protocol (Table 1) was performed on 5 subjects and 10 facial nerves. Imaging with both PETRA protocols was performed on 4 subjects.

\section{Imaging Analysis}

Two independent reviewers scored the visibility of the cisternal, canalicular, labyrinthine, geniculate, tympanic, and mastoid seg- 
ments of the facial nerve and the greater superficial petrosal nerve on each side of each subject on all series of default PETRA and 4-minute PETRA images. One reviewer was a neuroradiologist with 10 years' attending-level experience, and 1 reviewer was a postgraduate year-5 radiology resident completing a year-long neuroradiology mini-fellowship. To allow indirect comparison with a prior study that evaluated these facial nerve segments using a 3D inversion recovery fast spoiled gradient recalled (IR-FSPGR) sequence, ${ }^{1}$ we scored the signal intensity as follows: 0 , no signal; 1 , faint visualization; 2 , signal equivalent to that of the normal cer-

Table 1: Parameters for default and 4-minute PETRA sequences

\begin{tabular}{lll} 
& \multicolumn{1}{c}{ Default } & \multicolumn{1}{c}{ 4-Minute } \\
\hline TR 1 & $3.32 \mathrm{~ms}$ & $3.32 \mathrm{~ms}$ \\
TR 2 & $2250 \mathrm{~ms}$ & $2250 \mathrm{~ms}$ \\
TE & $0.07 \mathrm{~ms}$ & $0.07 \mathrm{~ms}$ \\
TI & $1300 \mathrm{~ms}$ & $2000 \mathrm{~ms}$ \\
TI 2 & $900 \mathrm{~ms}$ & $900 \mathrm{~ms}$ \\
Averages & 1 & 1 \\
Slice thickness & $0.9 \mathrm{~mm}$ & $0.9 \mathrm{~mm}$ \\
FOV & $300 \times 300$ & $300 \times 300$ \\
Matrix & $320 \times 320$ & $320 \times 320$ \\
Voxel size & $0.9 \times 0.9 \times 0.9 \mathrm{~mm}$ & $0.9 \times 0.9 \times 0.9 \mathrm{~mm}$ \\
Bandwidth & $400 \mathrm{kHz}$ & $400 \mathrm{kHz}$ \\
Flip angle & $6^{\circ}$ & $6^{\circ}$ \\
Radial views & 60,000 & 40,000 \\
Acquisition time & $5: 57 \mathrm{minutes}$ & $4: 05 \mathrm{minutes}$ \\
\hline
\end{tabular}
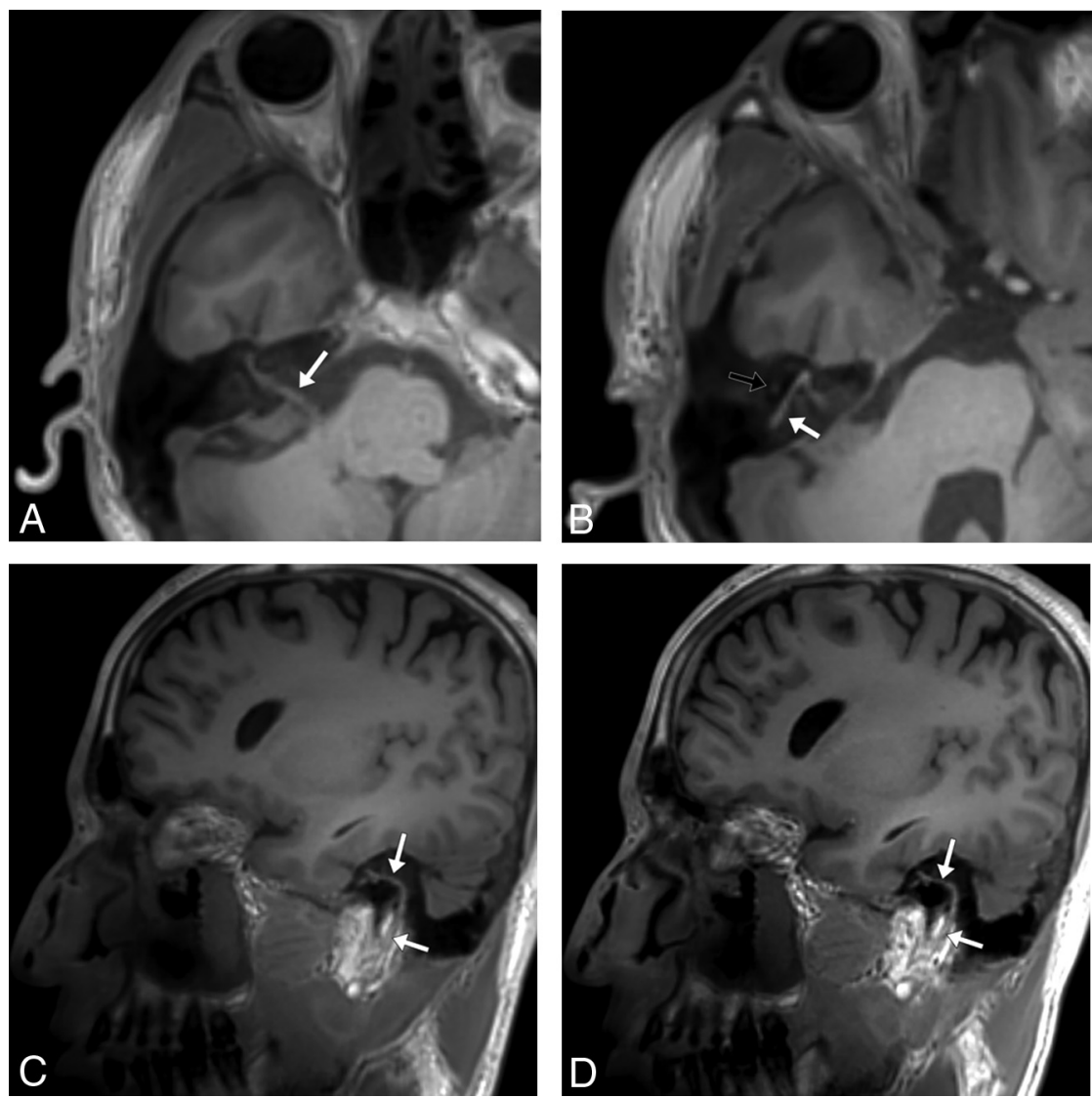

FIGURE. Normal right facial nerve in a 27-year-old healthy female volunteer. Axial oblique ( $A$ and $B$ ) and sagittal oblique $(C)$ noncontrast MR images obtained with the 4-minute PETRA protocol show the cisternal, canalicular, labyrinthine, geniculate, tympanic, and mastoid segments of the right facial nerve (white arrows) and a portion of an ossicle (black arrow). D, Sagittal oblique image obtained with the default PETRA protocol shows a slightly sharper but similar appearance to $C$. ebellum. The scored data from both reviewers were summed and averaged for each facial nerve segment for both the default PETRA and 4-minute PETRA images. Using sample size, means, and SDs, we calculated independent-sample $t$ tests to compare the signal intensity of the facial nerve on the 4-minute PETRA protocol images, as scored for this study, with the signal intensity of the facial nerve on the IR-FSPGR images, as scored in the study by Dehkharghani et al. ${ }^{1}$

To determine the SNR and CNR, in all 4 subjects in whom both protocols were performed, we drew ROIs on a single slice over the following structures of the default and 4-minute PETRA images: facial nerve tympanic segment, ossicle, middle ear cavity, mastoid, brain stem, and extracorporeal background region lateral to the temporal bone. The SNR and CNR were calculated as follows: SNR = Mean Signal Intensity of the Region/SD of Background Noise; CNR = SNR Region $1-$ SNR Region 2.

\section{RESULTS}

All evaluated segments of the facial nerve were visible by both reviewers (Figure) with a score of either 1 or 2 with $90.5 \%$ overall reviewer agreement. The signal intensity of all facial nerve segments was significantly greater for the 4-minute PETRA protocol images compared the IR-FSPGR images (Table 2).

SNR and CNR values were consistently similar or higher with the 4-minute PETRA protocol compared with the default PETRA protocol (Tables 3 and 4).

\section{DISCUSSION}

This study demonstrates that noncontrast UTE imaging with the PETRA sequence can provide visualization of the facial nerve from the brain stem to the stylomastoid foramen. Such visualization is not possible with more routine sequences currently used in skull base or internal auditory canal imaging. ${ }^{1,2}$ The PETRA sequence is a proved alternative to an IR-FSPGR sequence in pediatric brain imaging $^{8}$ and could perhaps similarly substitute for IR-FSPGR sequences in skull base imaging.

It is known that ultrashort T2 $(<1$ ms) MR signal can be detected in peripheral nerves and imaged. This signal originates from protons in myelin phospholipids ${ }^{5}$ and likely accounts, at least in part, for the consistent visibility of the facial nerve with the PETRA sequence. In addition, it is likely that artifacts related to the air/tissue interfaces in the temporal bone interfere with detection of signal from the facial nerve on more routine sequences. Minimization of airrelated susceptibility artifacts with the 
Table 2: Signal intensity comparison of PETRA and IR-FSPGR sequences as previously reported ${ }^{\mathrm{a}}$

\begin{tabular}{|c|c|c|c|c|}
\hline & Default PETRA & 4-Minute PETRA & $\begin{array}{c}\text { IR-FSPGR (from } \\
\text { Dehkharghani et al') }\end{array}$ & $\begin{array}{l}\text { P Value (4-Minute } \\
\text { PETRA vs IR-FSPGR) }\end{array}$ \\
\hline Cisternal & $1.89(0.31)$ & $2.00(0.00)$ & $0.86(0.42)$ & $<.001$ \\
\hline Canalicular & $1.86(0.36)$ & $2.00(0.00)$ & $0.83(0.44)$ & $<.001$ \\
\hline Labyrinthine & $1.79(0.42)$ & $1.85(0.37)$ & $0.88(0.39)$ & $<.001$ \\
\hline Geniculate & $2.00(0.00)$ & $2.00(0.00)$ & $1.03(0.22)$ & $<.001$ \\
\hline Tympanic & $1.96(0.19)$ & $2.00(0.00)$ & $0.95(0.32)$ & $<.001$ \\
\hline Superficial Petrosal & $2.00(0.00)$ & $2.00(0.00)$ & $0.96(0.30)$ & $<.001$ \\
\hline Mastoid & $2.00(0.00)$ & $2.00(0.00)$ & $1.01(0.30)$ & $<.001$ \\
\hline
\end{tabular}

a To allow direct comparison with the data published by Dehkharghani et al,' except for the $P$ values, we report data as mean (SD) of assigned signal intensity, in which each facial nerve segment was assigned a value of $0-2$ ( 0 , no detectable signal; 1 , faint visualization; 2 , signal equivalent to normal brain).

Table 3: Signal-to-noise ratio for default and 4-minute PETRA sequences

\begin{tabular}{|c|c|c|c|c|c|c|c|c|}
\hline & \multicolumn{2}{|c|}{ Subject C } & \multicolumn{2}{|c|}{ Subject D } & \multicolumn{2}{|c|}{ Subject F } & \multicolumn{2}{|c|}{ Subject G } \\
\hline & $\begin{array}{c}\text { Default } \\
\text { PETRA }\end{array}$ & $\begin{array}{c}\text { 4-Minute } \\
\text { PETRA }\end{array}$ & $\begin{array}{c}\text { Default } \\
\text { PETRA }\end{array}$ & $\begin{array}{l}\text { 4-Minute } \\
\text { PETRA }\end{array}$ & $\begin{array}{c}\text { Default } \\
\text { PETRA }\end{array}$ & $\begin{array}{l}\text { 4-Minute } \\
\text { PETRA }\end{array}$ & $\begin{array}{c}\text { Default } \\
\text { PETRA }\end{array}$ & $\begin{array}{c}\text { 4-Minute } \\
\text { PETRA }\end{array}$ \\
\hline Facial nerve & 125 & 165 & 113 & 159 & 88 & 140 & 99 & 173 \\
\hline Ossicle & 58 & 116 & 65 & 114 & 39 & 94 & 57 & 118 \\
\hline Middle ear & 5 & 51 & 31 & 75 & 36 & 83 & 14 & 59 \\
\hline Mastoid & 36 & 55 & 62 & 106 & 28 & 54 & 42 & 63 \\
\hline Brain stem & 168 & 281 & 155 & 290 & 151 & 277 & 176 & 300 \\
\hline
\end{tabular}

Table 4: Contrast-to-noise ratio for default and 4-minute PETRA sequences

\begin{tabular}{|c|c|c|c|c|c|c|c|c|}
\hline & \multicolumn{2}{|c|}{ Subject C } & \multicolumn{2}{|c|}{ Subject D } & \multicolumn{2}{|c|}{ Subject F } & \multicolumn{2}{|c|}{ Subject G } \\
\hline & $\begin{array}{c}\text { Default } \\
\text { PETRA }\end{array}$ & $\begin{array}{l}\text { 4-Minute } \\
\text { PETRA }\end{array}$ & $\begin{array}{c}\text { Default } \\
\text { PETRA }\end{array}$ & $\begin{array}{l}\text { 4-Minute } \\
\text { PETRA }\end{array}$ & $\begin{array}{l}\text { Default } \\
\text { PETRA }\end{array}$ & $\begin{array}{l}\text { 4-Minute } \\
\text { PETRA }\end{array}$ & $\begin{array}{l}\text { Default } \\
\text { PETRA }\end{array}$ & $\begin{array}{c}\text { 4-Minute } \\
\text { PETRA }\end{array}$ \\
\hline Facial nerve/mastoid & 89 & 111 & 51 & 43 & 75 & 69 & 57 & 88 \\
\hline Facial nerve/middle ear & 120 & 114 & 82 & 67 & 65 & 46 & 85 & 91 \\
\hline Ossicle/mastoid & 22 & 62 & 3 & 7 & 13 & 32 & 15 & 44 \\
\hline Ossicle/middle ear & 53 & 65 & 34 & 31 & 4 & 9 & 43 & 47 \\
\hline
\end{tabular}

UTE sequences ${ }^{6}$ may reduce such interference and may also contribute to the consistent visibility of the facial nerve with the PETRA sequence. Comparison of temporal bone imaging with multiple different UTE sequences could help elucidate the biophysical properties that allow imaging of the facial nerve through the temporal bone and help optimize the technique.

The default PETRA protocol runs nearly 6 minutes. We shortened the protocol to 4 minutes by reducing the radial acquisitions from 60,000 to 40,000. To recover SNR and CNR, we increased the first TI, which is used in the pointwise acquisition of central $k$-space data. ${ }^{6,8}$ Our increase of the TI from 1300 to $2000 \mathrm{~ms}$ would be expected to increase signal in the brain and nerve tissues at some expense to gray and white matter contrast. These parameter changes resulted in mild signal artifacts in the middle ear cavity, demonstrated by higher middle ear cavity SNR.

The major limitation of this study is that imaging was performed with a single MR imaging system on a small sample of healthy subjects and does not prove reproducibility on other MR imaging systems or in a clinical setting. In addition, the comparison of signal intensities between PETRA and IR-FSPGR sequences was made across studies performed by different researchers with different subjects and equipment.

\section{CONCLUSIONS}

A 3T MR imaging 4-minute noncontrast PETRA protocol enables visualization of the facial nerve from the brain stem, through the temporal bone, to the stylomastoid foramen. Use of the PETRA sequence, or other UTE sequences, should be considered in the
MR imaging evaluation of certain skull base tumors and perhaps other facial nerve and temporal bone pathologies.

Disclosures: Jeffrey P. Guenette-RELATED: Grant: American Society of Head and Neck Radiology. * Ravi Teja Seethamraju—UNRELATED: Employment: Siemens Medical Solutions USA, Inc.; Stock/Stock Options: Siemens Medical Solutions USA, Inc. Jayender Jagadeesan—RELATED: Grant: National Institutes of Health, Comments: through the National Institute of Biomedical Imaging and Bioengineering grant number P41EB015898*; UNRELATED: Board Membership: Navigation Sciences; Consultancy: Navigation Sciences; Grants/Grants Pending: Siemens Research Grant*; Patents (Planned, Pending or Issued): system for localizing deformable tumors*; Stock/ Stock Options: Navigation Sciences. Thomas C. Lee-RELATED: Grant: American Society of Head and Neck Radiology, Comments: This study was supported by the American Society of Head and Neck Radiology through the 2017 William N. Hanafee Research Grant*. *Money paid to the institution.

\section{REFERENCES}

1. Dehkharghani S, Lubarsky M, Aiken AH, et al. Redefining normal facial nerve enhancement: healthy subject comparison of typical enhancement patterns-unenhanced and contrast-enhanced spinecho versus $3 \mathrm{D}$ inversion recovery-prepared fast spoiled gradientecho imaging. AJR Am J Roentgenol 2014;202:1108-13 CrossRef Medline

2. Hong HS, Yi BH, Cha JG, et al. Enhancement pattern of the normal facial nerve at 3.0 T temporal MRI. Br J Radiol 2010;83:118-21 CrossRef Medline

3. Robson MD, Gatehouse PD, Bydder M, et al. Magnetic resonance: an introduction to ultrashort TE (UTE) imaging. J Comput Assist Tomogr 2003;27:825-46 CrossRef Medline

4. Naganawa S, Nakane $T$, Kawai $H$, et al. Visualization of middle ear ossicles in elder subjects with ultra-short echo time MR imaging. Magn Reson Med Sci 2017;16:93-97 CrossRef Medline

5. Horch RA1, Gore JC, Does MD. Origins of the ultrashort-T2 1H NMR 
signals in myelinated nerve: a direct measure of myelin content? Magn Reson Med 2011;66:24-31 CrossRef Medline

6. Grodzki DM, Jakob PM, Heismann B. Ultrashort echo time imaging using pointwise encoding time reduction with radial acquisition (PETRA). Magn Reson Med 2012;67:510-18 CrossRef Medline

7. Froidevaux R, Weiger M, Brunner DO, et al. Filling the dead-time gap in zero echo time MRI: principles compared. Magn Reson Med 2018; 79:2036-45 CrossRef Medline

8. Aida N, Niwa T, Fujii Y, et al. Quiet T1-weighted pointwise encoding time reduction with radial acquisition for assessing myelination in the pediatric brain. AJNR Am J Neuroradiol 2016;37: 1528-34 CrossRef Medline 